\title{
Gastric lanthanosis (lanthanum deposition) in an immunosuppressed patient that discontinued lanthanum carbonate
} seven years ago

\author{
Joana Ullmann ${ }^{1}$ and Andreas Erbersdobler ${ }^{1}$ \\ ${ }^{1}$ Universitätsmedizin Rostock
}

September 14, 2021

\begin{abstract}
A 72-year-old female used the oral phosphate binder lanthanum carbonate for 6 years, before discontinuing it after receiving a pancreas and kidney transplant. Now, 7 years after discontinuation, the patient developed bilious emesis. An upper gastrointestinal endoscopy showed an unspecific gastritis. Biopsies showed subepithelial crystalline deposits consistent with gastric lanthanosis.
\end{abstract}

\section{Introduction}

Lanthanum carbonate is one of the most potent phosphate binders. It binds dietary phosphate and forms insoluble complexes which are eliminated through the gastrointestinal tract. It is used to prevent elevation of serum phosphate especially in dialysis dependent patients. Patients treated with lanthanum carbonate show depositions predominantly in the gastric mucosa. Those depositions can be seen on gross examination as white spots, ringlike white discoloration, or diffuse white discoloration of the mucosa. Scanning electron microscopy and energy dispersive X-ray can be used to further identify lanthanum and phosphate deposition in the mucosa. The whitish depositions can be seen as early as three months after first taking lanthanum carbonate. They also seem to intensify over time. There is a positive correlation between the amount of lanthanum intake and its accumulation. The depositions seem to persist for a long time.

We herein report a case of gastric lanthanosis in a patient with a cessation of lanthanum carbonate over 7 years, supporting the concept of permanent depositions. This could suggest that the depositions are irreversible or at least persist for a long time. They also seem to lead to symptoms even years after cessation. Clinicians should be aware of this fact and specifically look for specific endoscopic changes and take biopsies if they have a patient with epigastric discomfort and a history of lanthanum medication.

\section{Case report}

A 72-year-old female patient was admitted to the nephrology department due to inadequately controlled arterial hypertonus, non-dipper type, and declined function of a renal transplant. The patient has chronic renal failure secondary to type 1 diabetes. The patient began hemodialysis in 2007. She was treated with the phosphate binder lanthanum carbonate for 6 years, with a daily dose of $2000 \mathrm{mg} /$ day orally. The patient received a combined pancreas and renal transplant in 2013. After the transplantation, immunosuppressive therapy with Tacrolimus $(0,5 \mathrm{mg} / \mathrm{d})$ and Mycophenolat $(1000 \mathrm{mg} / \mathrm{d})$ was started, and lanthanum carbonate was no longer taken. The pancreas transplant was explanted due to a T-cell-mediated rejection.

During the inpatient stay the patient developed bilious emesis without enteritis and without signs for an infectious genesis. A sonography of the abdomen was performed. It showed an empty stomach and distended, 
up to $31 \mathrm{~mm}$, small bowel filled with fluids. An abdominal radiograph showed the bowel to be filled with stool and little gas with few air-fluid-levels in the central and right upper abdomen. No free abdominal gas was seen. The patient showed signs of coprostasis, and the diagnosis of a subileus was suspected. An upper gastrointestinal endoscopy was performed the next day. A reddened mucosa and hematin fragments were seen and a hemorrhagic gastritis was described. Biopsies were taken from the antrum and corpus of the stomach. Histologic evaluation of gastric biopsies showed near normal antral mucosa without significant inflammation. Notably, helicobacter-associated gastritis was not present. Gastric biopsies from the fundus displayed patchy fibrotic areas, preferentially underneath and between the foveolar epithelium. Here, histiocytes containing crystalline material were present without significant infiltrates of lymphocytes, plasma-cells, or granulocytes (Fig. 1 and 2). The specialized fundic glands were well preserved. The findings were considered typical for lanthanum-induced gastropathy.

After an enema the gastrointestinal symptoms were reduced. During the inpatient stay the arterial hypertonus was successfully managed and thus the edema and proteinuria could be reduced.

\section{Discussion}

To reduce hyperphosphatemia in dialysis dependent patients there are different oral phosphate binders available. As compared with other phosphate binders, Lanthanum carbonate does not show an increased risk for all-cause mortality or malignancy while maintaining a similar effect of reducing serum phosphate levels. However, $17.8 \%$ of patients showed gastrointestinal symptoms that required hospitalization. Unspecific gastrointestinal symptoms, like emesis, are the most common adverse effects. The oral bioavailability is $0.001 \%$ and is mostly eliminated through the gastrointestinal tract where it forms insoluble complexes with ingested phosphate. Since 2015 numerous case reports and a few studies showed an association with lanthanum carbonate intake and lanthanum phosphate deposition especially in the gastric mucosa. Nevertheless, the accumulation mechanism is not entirely understood. Lanthanum carbonate is soluble at resting gastric $\mathrm{pH}-$ levels which could be a way of penetrating the gastric mucosa. Chronic kidney disease patients also have a disruption of the epithelial tight junctions in the gastrointestinal tract which leads to a higher permeability for substances like lanthanum. Lanthanum carbonate is radiopaque. In abdominal CT, a high-density linear appearance (HD-LA), can be found in $60 \%-79 \%$, and is seen as a correlate for gastric lanthanum depositions. Yabuki et al. suggest using the HD-LA as a screening criterion. Whereas Namie et al. described a continuing HD-LA in CT after 8 months of discontinuation, our patient didn't show radiopaque objects in an abdominal radiograph, but radiographs may not be as sensitive to slight lanthanum depositions as a CT might be. Further studies are needed to investigate if abdominal radiographs and CTs can be used as a screening method. Endoscopically the depositions appear as whitish mucosal lesions that vary in shape and size. Although Shitomi et al. found that those distinctive endoscopic observations might be difficult to detect in a case with slight depositions. This is why Iwamuro et al. suggest taking biopsies also in cases without an endoscopic correlate because they found lanthanum depositions by histology in endoscopically normal mucosa.

The histologic correlate to the whitish discoloration are histiocytes containing brownish foreign bodies in the lamina propria. In an early stage of deposition, the macrophages are arranged singly or in small clusters, whereas in later stages they diffusely infiltrate the mucosa. The macrophages in our specimen showed a diffuse infiltration pattern, which fits with a later stage accumulation. Nakamura et al. suggest that M2-polarised macrophages play a crucial role for the clearance of lanthanum. Lanthanum is likely to be resistible to intracellular digestion, so histiocytic activation with following tissue damage is conceivable. The endoscopic features show a significant relationship with the degree of histiocytic infiltration. Hattori et al. found that the histiocytic infiltration is specific for lanthanum deposition. The daily dose of lanthanum carbonate positively correlates with lanthanum deposition in the mucosa. Various histopathological changes are described with lanthanum depositions. Most often metaplastic changes, regenerative changes, foveolar hyperplasia, and chromic or active inflammation are described. Nishida et al. found no association between the range of atrophic changes and lanthanum depositions. Our patient also showed no atrophic changes in the mucosa, which would be concordant with Nishidas observations. Haratake et al. and Valika et 
al. found that preformed ulcers as well as underlying pathologies induced by NSARs aggravate the entry of lanthanum.As those mucosal changes are often described with a helicobacter pylori positive gastritis, several authors looked for a relationship between helicobacter infection and lanthanum deposition. Namie et al. suggest that a helicobacter pylori infection may aggravate lanthanum entry through the compromised mucosal barrier. Shitomi et al. did not find helicobacter pylori infection in patients with a lanthanosis and propose that a helicobacter infection might prevent accumulation of lanthanum in the mucosa through a higher $\mathrm{pH}$ and a disturbed histiocytic infiltration in the setting of an inflammation. Iwamuro et al. suggest that the gastric body is predominantly affected by lanthanum deposition, which was also true for our patient, because of the prolonged contact time of lanthanum with the mucosa.

Another reason why it is important that endoscopist should know about gastric lanthanosis is that it can endoscopically imitate early gastric cancer. Yabuki et al. even described squamous cell carcinoma associated with lanthanum deposition in a rat model. They also found that other mucosal lesions such as chronic inflammation and intestinal metaplasia were more frequent in mucosa with lanthanum depositions. So, they conclude that lanthanum could indirectly induce metaplasia. Tabushi et al. described the first case of early gastric cancer in association with gastric lanthanosis. Shitomie et al. and Takatsuna et al. described gastric cancer in gastric lanthanosis patients, but they found less or any lanthanum depositions in the neoplasia itself. They suggest that the neoplastic mucosa has a malfunctioning absorption mechanism which leads to lesser lanthanum deposition in that area. With this in mind it is crucial to know how those depositions develop over time and if they are reversible. Lanthanum depositions seem to gradually expand over time, while taking lanthanum carbonate, in humans as well as in a rat model. Whilst Rothenberg et al. observed a reduction in symptoms and histological findings after cessation of lanthanum carbonate, Awad et al. found only a reduction of symptoms. Namie et al. and Hoda et al. described persisting depositions after cessation. Hoda et al. reported one patient that received a kidney transplant and discontinued lanthanum carbonate. What this patient has in common with our patient is the discontinuation of lanthanum carbonate due to kidney transplant and continuing lanthanum deposition in the gastric mucosa. We found no other cases in the literature that described a long cessation of lanthanum carbonate with lanthanum deposits. A limitation of our case report is that we have no preceding endoscopies or biopsies, so we do not know how the lanthanum deposition changed over time. We think that the immunosuppressants our patient takes could be involved in the long duration of the deposition. As Tacrolimus and Mycophenolat inhibit mainly lymphocytes, but also macrophage immune response one could postulate that this leads to a prolonged clearance of lanthanum. But neither the accumulation-, nor the clearance mechanism are understood, so we can only speculate at this moment.

\section{Conclusion}

It is important to be aware of the diagnosis of gastric lanthanosis and to keep in mind the long persistence of lanthanum carbonate in the gastric mucosa, which can be detected even years after cessation of lanthanum intake.

\section{Informed Consent}

Written informed consent was obtained from the patient for publication of this case report and accompanying images.

\section{Conflict of Interest}

The authors have no conflict of interests to disclose.

\section{Authorship List}

Joana Maria Ullmann(joanaMaria.ullmann@med.uni-rostock.de; Universitätsmedizin Rostock, Institute of Pathology, Strempelstraße 14, 18055 Germany): Drafting of the article and substantial contributions to the conception of the work.

Prof. Dr. med. Andreas Erbersdobler(andreas.erbersdobler@med.uni-rostock.de; Universitätsmedizin 
Rostock, Institute of Pathology, Strempelstraße 14, 18055 Germany): diagnostic responsibility, critical revision of the article and substantial contributions to the conception of the work.

\section{References}

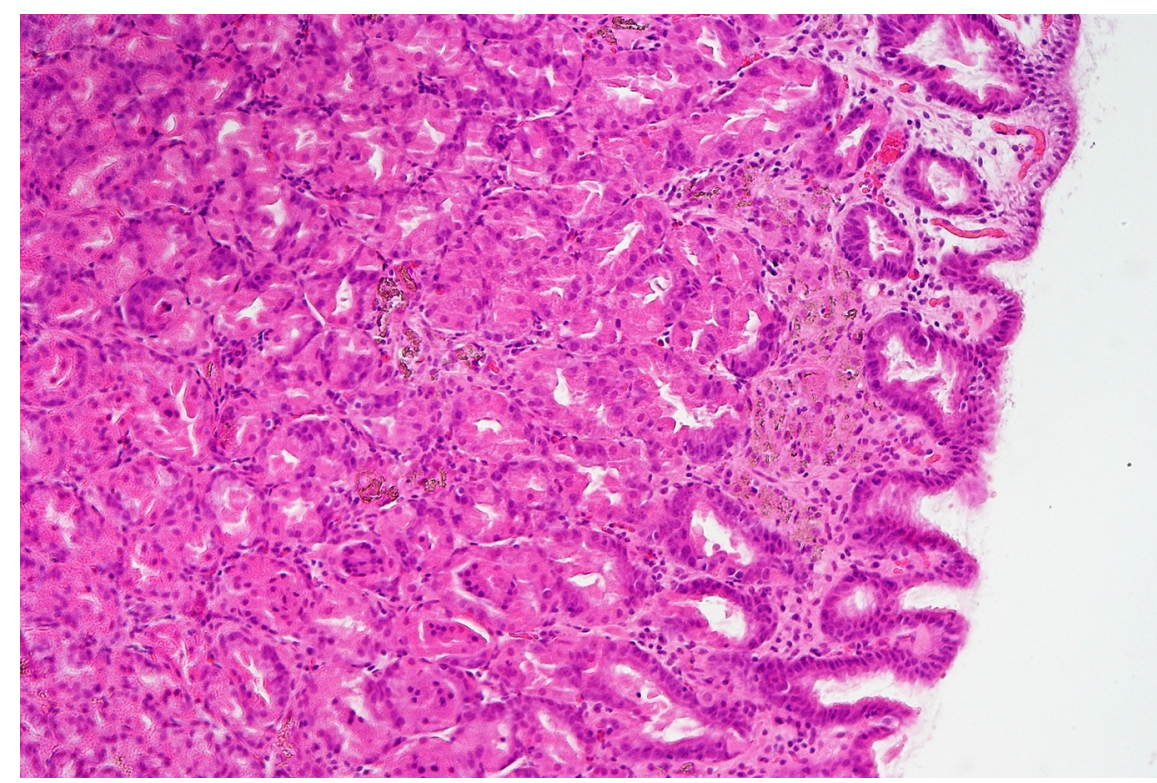

Fig. 1 Hematoxylin\&Eosin-stained sections from the fundic mucosa showing lanthanum-induced gastropathy (original magnifications: $\mathrm{x} 100$ )

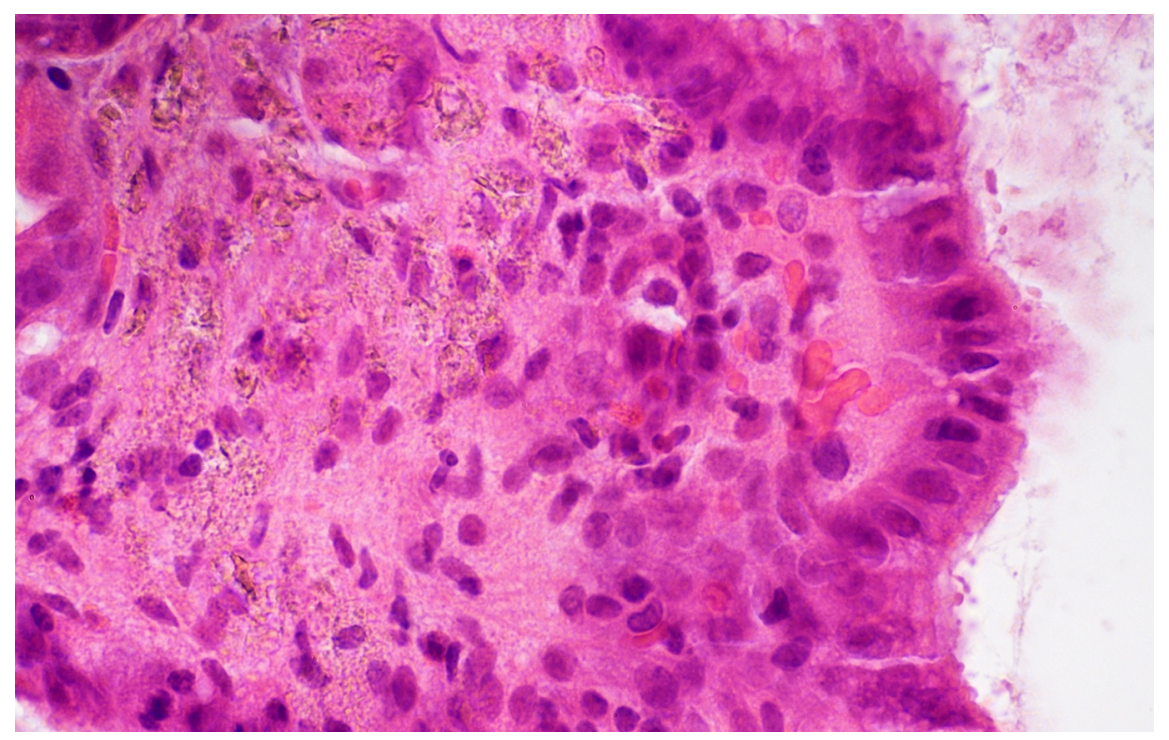

Fig. 2 Hematoxylin\&Eosin-stained sections from the fundic mucosa showing lanthanum-induced gastropathy (original magnifications: $\mathrm{x} 400$ ) 